\title{
Relación entre estrategias de aprendizaje, elección vocacional, rendimiento académico y personalidad predominante en los alumnos del CEPRE-UNMSM - Ciclo Extraordinario 2013-2014
}

\author{
Relationship between learning strategies, career choice, academic achievement and \\ personality prevalent in students CEPRE-San Marcos - Extraordinary Cycle 2013-2014. \\ Hilda Chávez Ch., Juan Morocho S., César Alvites R., José Vega G., Jesús Ruelas S., Christian Espinoza M., \\ Juan Gómez Ch., Fabiana Santiago A. \\ Universidad Nacional Mayor de San Marcos, Lima, Perú \\ (RECIBIDO 08/10/2015, AcEPTADO 10/02/2016)
}

\begin{abstract}
RESUMEN
Se planteó el desarrollo de una investigación no experimental, descriptivo-correlacional y transeccional. Se inicia con la descripción general de cada variable de estudio: estrategias de aprendizaje, elección vocacional, rendimiento académico y personalidad predominante en los alumnos del CEPRE-UNMSM - Ciclo Extraordinario 2013-2014, trabajando las relaciones entre las variables de estudio. Se utilizó la escala de estrategias de aprendizaje elaborada por Román y Gallego, el test de autoevaluación vocacional de Holland, encuesta psicopedagógica elaborada por los autores del estudio (Chávez y Ruelas, 2012) y rendimiento académico de los estudiantes en los exámenes del CEPRE - UNMSM. Los resultados se han sometido al análisis estadístico correspondiente utilizando MS - Excel y la versión 20 del SPSS.

Se obtuvo como resultado que las estrategias de aprendizaje más utilizadas por los alumnos son repetición y relectura $(80.88 \%)$. En la elección vocacional la carrera profesional que tiene mayor demanda es Medicina Humana.

En la correlación de estrategias de aprendizaje y elección vocacional, las dos variables son independientes, no están asociadas, por lo tanto no rechazamos la hipótesis nula. En la correlación estrategias de aprendizaje y rendimiento académico, las variables son independientes. En correlación estrategias de aprendizaje y personalidad, las dos variables son independientes, no están asociadas, por lo tanto no rechazamos la hipótesis nula. Los resultados cuantitativos nos indican, en su contrastación a nivel correlacional, que no existe una relación directa significativa entre las estrategias de aprendizaje con el rendimiento académico, personalidad y elección vocacional.
\end{abstract}

Palabras clave: Rendimiento académico, aprendizaje, vocación 


\begin{abstract}
The development of a non-experimental, descriptive and correlational research transeccional was raised; which begins with a general description of each study variable: learning strategies, career choice, academic achievement and personality prevalent in students CEPRESan Marcos - Extraordinary Cycle 2013 - 2014, working relationships between the study variables. San Marcos - scale learning strategies developed by Roman and Gallego, the test of vocational self Holland, psychology survey conducted by the study authors (Chavez and Ruelas, 2012) and academic performance of students in examinations CEPRE was used. The results were submitted to the appropriate statistical analysis using MS - Excel and SPSS version 20. It resulted that more learning strategies used by students is repetition and reinterpretation $(80.88 \%)$. In vocational career choice with greater demand Human Medicine. Correlation strategies in learning and career choice, the two variables are independent, they are not associated, so do not reject the null hypothesis.

In Correlation learning strategies and personality, the two variables are independent, they are not associated, so do not reject the null hypothesis. The quantitative results indicate a correlation in its contrast level that there is no significant direct relationship between learning strategies with academic performance, personality and career choice.
\end{abstract}

Keywords: Academic performance, learning, vocation

\title{
INTRODUCCIÓN
}

El desarrollo de esta investigación permitió establecer las fortalezas y debilidades de los alumnos del CEPRE-UNMSM - Ciclo Extraordinario 2013-2014, con relación a las estrategias de aprendizaje, elección vocacional, rendimiento académico y su personalidad predominante. Asimismo, nos permitió describir estas variables en función del tipo de familia (nuclear, extendida, monoparental y fusionada), tipo de institución educativa (pública y privada) y lugar de procedencia.

En la metodología se ha considerado la aplicación de la escala de estrategias de aprendizaje (ACRA), elaborada por Román y Gallego (1994), con el objetivo de identificar las estrategias de aprendizaje más frecuentes utilizadas por los estudiantes cuando están asimilando la información contenida en un texto, en un artículo, en unos apuntes o cuando están estudiando. El procesamiento de la información es la base del aprendizaje y requiere, por ello, utilizar estrategias cognitivas adecuadas. El ACRA contiene cuatro escalas que evalúan las estrategias y cada una contiene lo siguiente:

- Siete estrategias de adquisición de la información.

- Trece estrategias de codificación de la información.

- Cuatro estrategias de recuperación de información.

- Nueve estrategias de apoyo al procesamiento. 
Se pueden aplicar y valorar de forma independiente. Sus resultados, global o factorialmente considerados, permiten informar, diagnosticar y orientar a los alumnos a llevar a cabo trabajos de investigación experimental y correlacional.

Para la elección vocacional, se usó como instrumento el test de autoevaluación vocacional de Holland, el cual relaciona la personalidad predominante con la carrera elegida por el estudiante. Para él, la elección de una carrera sería una extensión de la personalidad y un intento por implantar, dentro del contexto de la vida laboral, el estilo particular de comportamiento. En este sentido, los intereses vocacionales son sencillamente otro aspecto de la personalidad. Clasifica seis tipos de personalidad, correspondientes a seis tipos de ambientes laborales (convencionales, realistas, investigativos, artísticos, sociales y emprendedores). Según Holland, es muy importante la relación entre tipo de personalidad y ambiente, ya que la conducta de las personas está determinada por una interacción entre sus características de personalidad y las características y exigencias del ambiente en que se desenvuelven.

Desde el punto de vista técnico-científico, esta investigación contribuye a enriquecer los conocimientos con relación a las variables de estudio. Por un lado, fortalecer la validez, confiabilidad y objetividad de los instrumentos psicométricos utilizados. Por otro lado, posibilita que los interesados desarrollen e implementen programas de intervención, a fin de detectar las carencias a nivel cognitivo (estrategias de aprendizaje) de los adolescentes y, a la vez, fortalecer la toma de decisiones con respecto a la carrera a elegir de estos, estableciendo la relación entre su personalidad predominante y la elección vocacional. Apoya de esta manera a su mejor desarrollo académico, personal y familiar. De esta manera no solo se beneficia a la Universidad, sino también a las instituciones educativas de Lima, por UGEL o proporcionar los alcances a las autoridades del Ministerio de Educación.

\section{OBJETIVOS}

\section{Objetivo general}

Conocer la relación existente entre estrategias de aprendizaje, elección vocacional, rendimiento académico y personalidad predominante en los alumnos del CEPREUNMSM - Ciclo Extraordinario 2013-2014.

\section{Objetivos específicos}

1. Describir las estrategias de aprendizaje, elección vocacional, rendimiento académico y personalidad predominante en los alumnos del CEPRE-UNMSM - Ciclo Extraordinario 2013-2014.

2. Establecer la relación entre estrategias de aprendizaje y elección vocacional en los alumnos del CEPRE-UNMSM - Ciclo Extraordinario 2013-2014. 
3. Determinar la relación entre estrategias de aprendizaje y rendimiento académico en los alumnos del CEPRE-UNMSM - Ciclo Extraordinario 2013-2014.

4. Determinar la relación entre estrategias de aprendizaje y personalidad predominante en los alumnos del CEPRE-UNMSM - Ciclo Extraordinario 2013-2014.

\section{Hipótesis general}

Existe relación entre estrategias de aprendizaje, elección vocacional, rendimiento académico y personalidad predominante en los alumnos del CEPRE-UNMSM Ciclo Extraordinario 2013-2014.

\section{Hipótesis específicas}

1. Existe relación directa entre estrategias de aprendizaje y elección vocacional en los alumnos del CEPRE-UNMSM - Ciclo Extraordinario 2013-2014.

2. Entre las estrategias de aprendizaje y el rendimiento académico de los alumnos del CEPRE-UNMSM - Ciclo Extraordinario 2013-2014 existe relación directa.

3. Existe relación directa entre estrategias de aprendizaje y personalidad predominante en los alumnos del CEPRE-UNMSM - Ciclo Extraordinario 2013-2014.

\section{MÉTODO}

Se planteó una investigación no experimental, descriptivo-correlacional y transeccional; la cual se inicia con la descripción general de cada variable de estudio (estrategias de aprendizaje, elección vocacional, rendimiento académico y personalidad predominante), luego se procedió a presentar las variables descritas considerando tipo de colegio (público y privado), tipo de familia y lugar de procedencia. Por último, se presentarán las relaciones entre las variables de estudio.

\section{Población y muestra}

Constituida por los alumnos del CEPRE-UNMSM - Ciclo Extraordinario 20132014.

\section{Recolección e interpretación de datos}

Para el desarrollo del presente estudio, se utilizaron los siguientes instrumentos psicométricos:

- Escala de estrategias de aprendizaje, elaborada por Román y Gallego (1994), 
con el objetivo de identificar las estrategias de aprendizaje más frecuentes utilizadas por los estudiantes cuando están asimilando la información contenida en un texto, en un artículo, en unos apuntes o cuando están estudiando.

- Elección vocacional, usando como instrumento el test de autoevaluación vocacional de Holland, el cual relaciona la personalidad predominante con la carrera elegida por el estudiante.

- Encuesta psicopedagógica elaborada por los autores del estudio (Chávez y Ruelas, 2012).

- Rendimiento académico de los estudiantes en los exámenes del CEPRE UNMSM.

\section{RESULTADOS}

\section{Objetivo específico}

1.- Describir las estrategias de aprendizaje, elección vocacional, rendimiento académico y personalidad predominante en los alumnos del CEPRE-UNMSM Ciclo Extraordinario 2013-2014.

\section{Descripción de las estrategias de aprendizaje}

Tal como se puede apreciar en la siguiente tabla, las estrategias de aprendizaje más utilizadas por los alumnos del Ciclo Extraordinario 2013 - 2014 son: repetición y relectura $(80.88 \%)$ seguida de comprensión (78\%) y motivación intrínseca (77.88\%).

Asimismo, las estrategias de aprendizaje menos utilizadas por los alumnos son control de ansiedad (63\%), selección y organización (63.75\%) y horario y plan de trabajo $(64.13 \%)$. 
Tabla 1-1 Estrategias de aprendizaje utilizadas por los alumnos del Ciclo Extraordinario 20132014, CEPRE - UNMSM

\begin{tabular}{|l|c|}
\hline ESTRATEGIAS & $\%$ \\
\hline Selección y organización & 63.75 \\
\hline Subrayado & 67.31 \\
\hline Conciencia de la funcionalidad de las estrategias & 69.60 \\
\hline Estrategias de elaboración & 72.17 \\
\hline Planificación y control en situaciones de evaluación & 73.30 \\
\hline Repetición y relectura & $\mathbf{8 0 . 8 8}$ \\
\hline Motivación intrínseca & 77.88 \\
\hline Control de la ansiedad & 63.00 \\
\hline Condiciones contra distractores & 69.75 \\
\hline Apoyo social & 77.05 \\
\hline Horario y plan de trabajo & 64.13 \\
\hline Comprensión & 78.00 \\
\hline Hábitos de estudio & 68.50 \\
\hline
\end{tabular}

El mayor o menor uso de las diferentes estrategias de aprendizaje evaluadas por el ACRA se pueden apreciar mejor en la figura siguiente:

Figura 1-1 "Representación gráfica porcentual de las Estrategias de Aprendizaje utilizadas por los alumnos del Ciclo Extraordinario 2013-2014. CEPRE - UNMSM

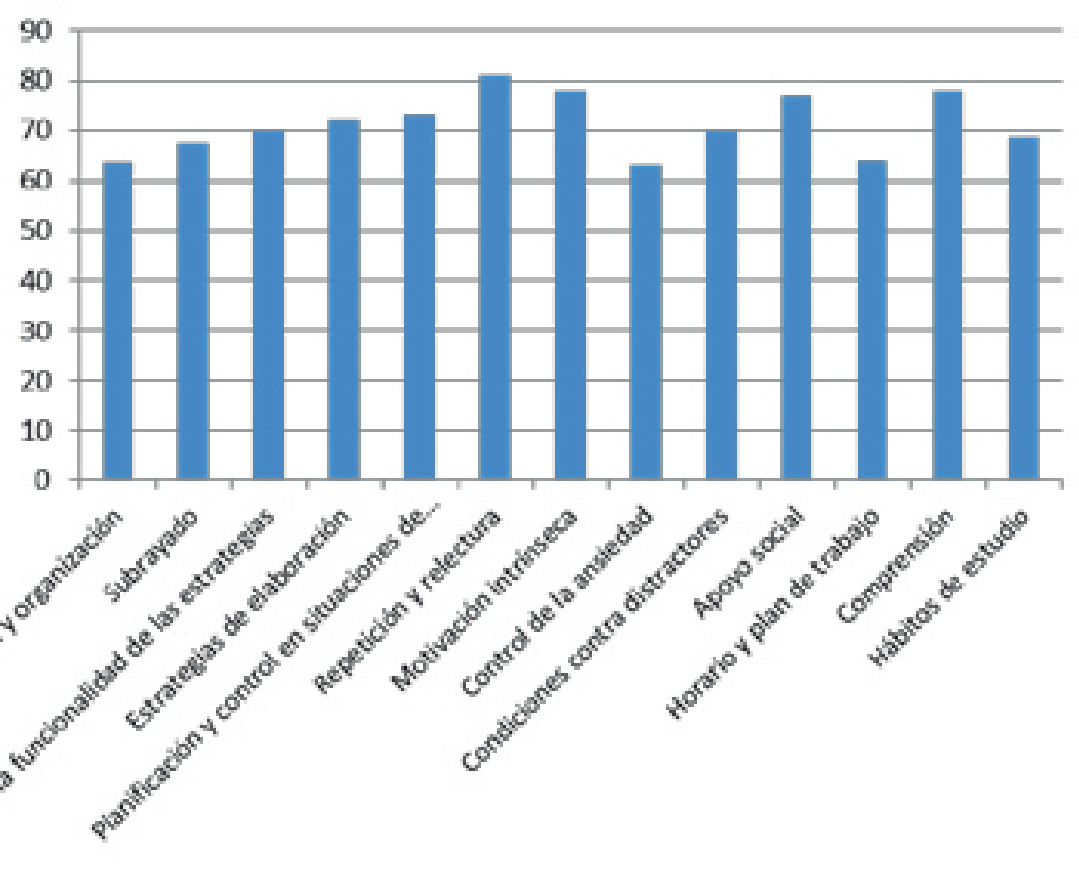




\section{Descripción de la elección vocacional}

Tal como se puede apreciar en las tablas $1-2 \mathrm{a}$ y $1-2 \mathrm{~b}$ las carreras profesionales que tienen mayor demanda son Medicina Humana (18.3\%), Ingeniería Industrial (13.5\%) y Administración (10.3\%). Asimismo, se puede observar claramente que son varias las carreras profesionales que tienen menor demanda, entre ellas mencionaremos a Literatura, Química y Sociología -entre otras- cada una de ellas con una demanda del $0.2 \%$.

Las demandas de los alumnos, por las diferentes carreras profesionales se pueden apreciar con mayor claridad con una representación gráfica (ver la Figura 1-2).

Tabla 1-2a Demanda porcentual de las carreras profesionales a las que postulan los alumnos del Ciclo Extraordinario 2013-2014 del CEPRE - UNMSM

\begin{tabular}{|c|c|c|}
\hline CARRERA A LA QUE POSTULA & FRECUENCIA & DORCENTANE \\
\hline AOMINISTRAOÓN & 43 & 10,3 \\
\hline ADMINISTRACÓN DE NEGOCIOS INTERNACIONALES & 35 & 7,5 \\
\hline ADMINISTRAOÓN OE TURISMO Y HOTELERIA & 7 & 1,5 \\
\hline ANTROPOLOGIA & 1 & 0.2 \\
\hline ARQUEOLOGÍA: & 3 & 0,6 \\
\hline AUDITORIA EMPRESARLAL & 3 & 0,6 \\
\hline OENCAS BIOLÓGICAS & 3 & 0,6 \\
\hline OENOAS FISICAS & 1 & 0,2 \\
\hline OENCIAS POLÍTICAS & 1 & 0,2 \\
\hline OENOAS SCOALES & 1 & 0,2 \\
\hline COMUNICACIÓN AUDIOVISUAL & 1 & 0.2 \\
\hline COMUNICACOÓN SOCIAL & 11 & 2,4 \\
\hline CONTAEIUDAO & 27 & 5,8 \\
\hline DERECHO & 32 & 6,9 \\
\hline ECONOMIA & 15 & 3.2 \\
\hline ECONOMIA INIERNACIONAL & 2 & 0,4 \\
\hline coUCACIÓN FISICA & 1 & 0,2 \\
\hline EOUCACIÓN SECUNDARIA & 1 & 0,2 \\
\hline ENFERMERIA & 1 & 0.2 \\
\hline FARMACIA T UOQUIMICA & 2 & 0,4 \\
\hline GENÉTICAY YIOTECNOLOGIA & 3 & 0,6 \\
\hline GEOGRAFIA & 1 & 0.2 \\
\hline GESTIÓN TRIBUTARIA & 3 & 0,6 \\
\hline INOENIERIA OVIL & 12 & 2,6 \\
\hline INGENIERIA DE MINAS & 9 & 1,9 \\
\hline INGENIERIA DE SISTEMAS & 19 & 4.1 \\
\hline INGENIERIA DE SOFTWARE & s & 1,1 \\
\hline INGENIERIA DE TELECOMUNICACIONES & 2 & 0,4 \\
\hline INGENIERIA EIÉCTRICA & 4 & 0,9 \\
\hline INGENIERIA ELECTRÓNICA & s & 11 \\
\hline INGENIERIA GEOGRÁFICA & 2 & 0,4 \\
\hline INGENIERIA GEOLOGICA & 3 & 1,7 \\
\hline INGENIERIA INDUSTRIAL & 63 & 13.5 \\
\hline INGENIERIA METALỦRGICA & 1 & 0.2 \\
\hline INGENIERIA QUIMICA & 2 & 0,4 \\
\hline INVESTIGAOÓN OPERATIVA & 1 & 0,2 \\
\hline
\end{tabular}


Tabla 1-2b Demanda porcentual de las carreras profesionales a las que postulan los alumnos del Ciclo Extraordinario 2013-2014 del CEPRE - UNMSM

\begin{tabular}{|l|r|r|}
\hline CARRERA A LA QUE POSTULA & FRECUENCIA & \multicolumn{1}{l|}{ PORCENTAJE } \\
\hline LITERATURA & 1 & 0,2 \\
\hline MEDICINA HUMANA & 85 & 18,3 \\
\hline MEDICINA VETERINARIA & 6 & 1,3 \\
\hline NUTRICIÓN & 2 & 0,4 \\
\hline OBSTETRICIA & 3 & 0,6 \\
\hline ODONTOLOGIA & 11 & 2,4 \\
\hline PSICOLOGIA & 13 & 2,8 \\
\hline QUIMICA & 1 & 0,2 \\
\hline RADIOLOGIA & 1 & 0,2 \\
\hline SOCIOLOGIA & 1 & 0,2 \\
\hline TECNOLOGIA MÉDICA & 1 & 0,2 \\
\hline TECNOLOGIA MÉDICA LABORATORIO & 2 & 0,4 \\
\hline TERAPIA FISICA & 1 & 0,2 \\
\hline TERAPIA OCUPACIONAL & 1 & 0,2 \\
\hline
\end{tabular}

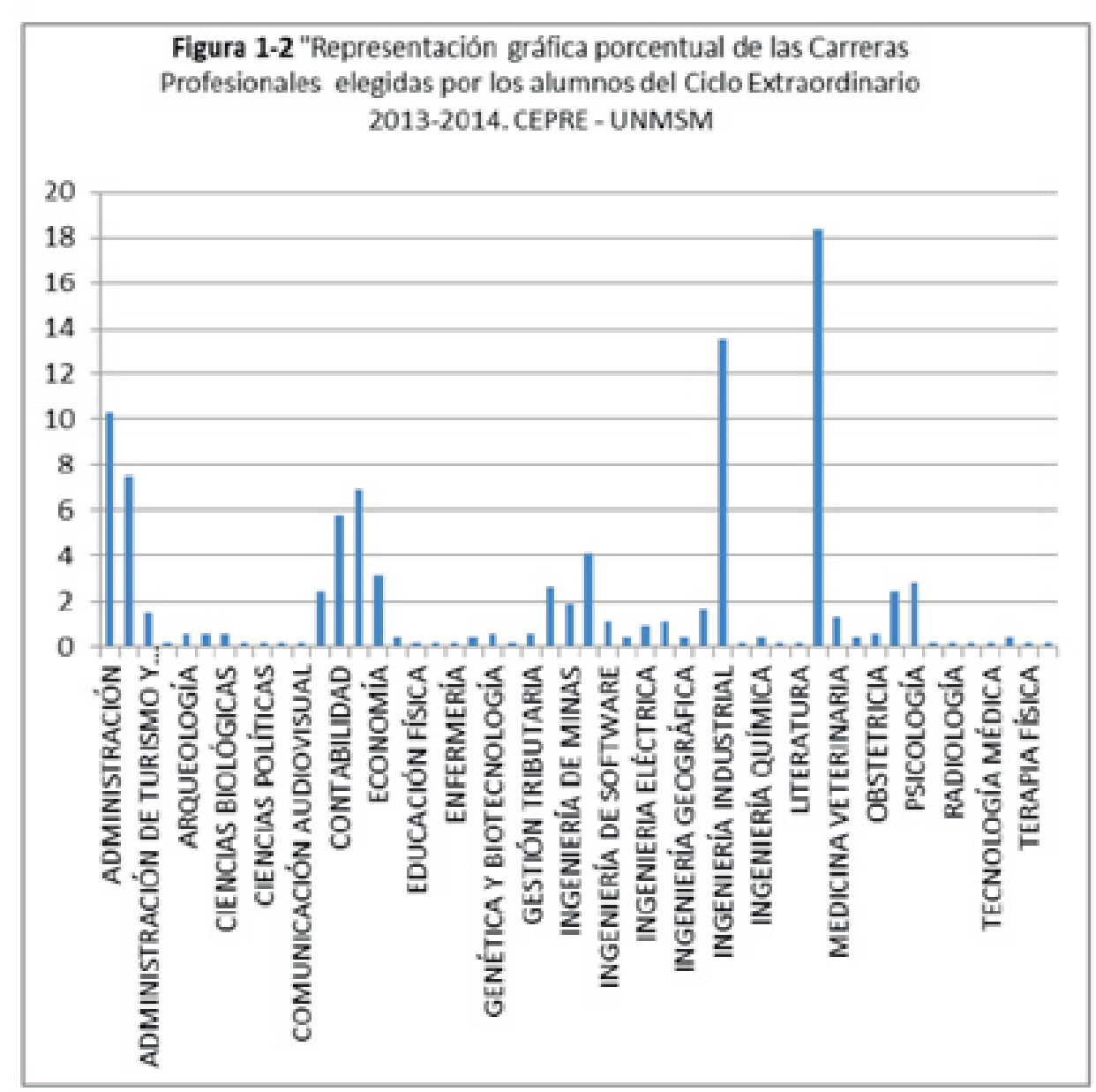




\section{Descripción del rendimiento académico}

Tal como se puede apreciar en la tabla 1-3, el rendimiento académico promedio de los alumnos del Ciclo Extraordinario 2013-2014 del CEPRE - UNMSM es de 649.46 puntos, con una desviación estándar de 297.189.

Tabla 1-3 Rendimiento académico de los alumnos del Ciclo Extraordinario 2013-2014. CEPREUNMSM

\begin{tabular}{|l|c|c|}
\hline \multirow{2}{*}{$\mathrm{N}$} & Válidos & 397 \\
\cline { 2 - 3 } & Perdidos & 0 \\
\hline Media & 649,46 \\
\hline Mediana & 613,50 \\
\hline Moda & 644 \\
\hline Desv. típ. & 297,189 \\
\hline Asimetría &, 498 \\
\hline Error típ. de asimetría &, 122 \\
\hline Curtosis &,- 097 \\
\hline Error típ. de curtosis &, 244 \\
\hline Mínimo & 34 \\
\hline Máximo & 1594 \\
\hline
\end{tabular}

Estos resultados se pueden apreciar mejor en la figura 1-3

Figura 1-3: "Representación gráfica del Rendimiento Académico de los alumnos del Ciclo Extraordinario 2013-2014. CEPRE-UNMSM"

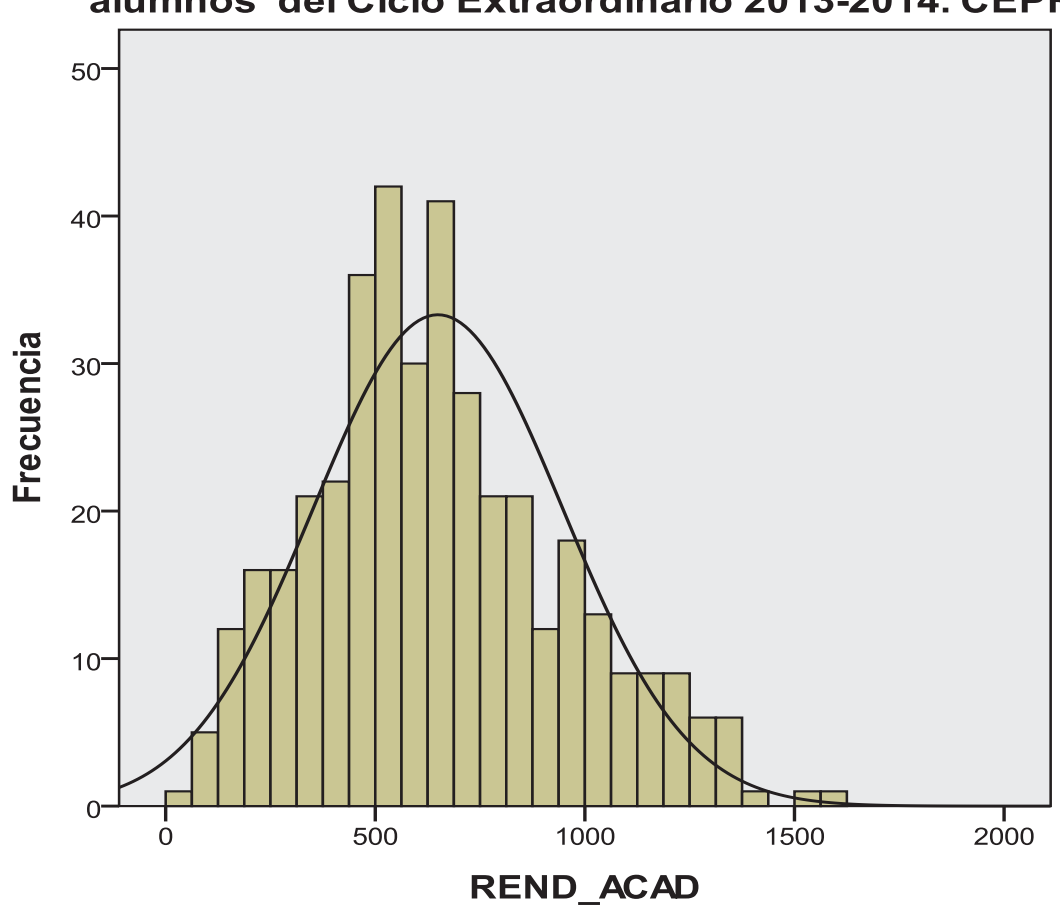


$\mathrm{Al}$ efectuar un análisis del rendimiento académico, considerando la variable sexo (ver Tabla 1-4), encontramos que las mujeres tienen un rendimiento académico promedio (651.57 puntos) mayor que el de los varones (645.97 puntos).

Tabla 1-4 Rendimiento académico de los alumnos del Ciclo Extraordinario 2013-2014. CEPREUNMSM"

\begin{tabular}{|c|c|c|c|}
\hline SEXO & N & Media & Desv. típ. \\
\hline Varones & 149 & 645,97 & 327,570 \\
\hline Mujeres & 248 & 651,57 & 278,019 \\
\hline Total & 397 & 649,46 & 297,189 \\
\hline
\end{tabular}

Para apreciar mejor estos resultados podemos efectuar una representación gráfica de ellos (Ver Figuras 1-4 a y 1-4 b).

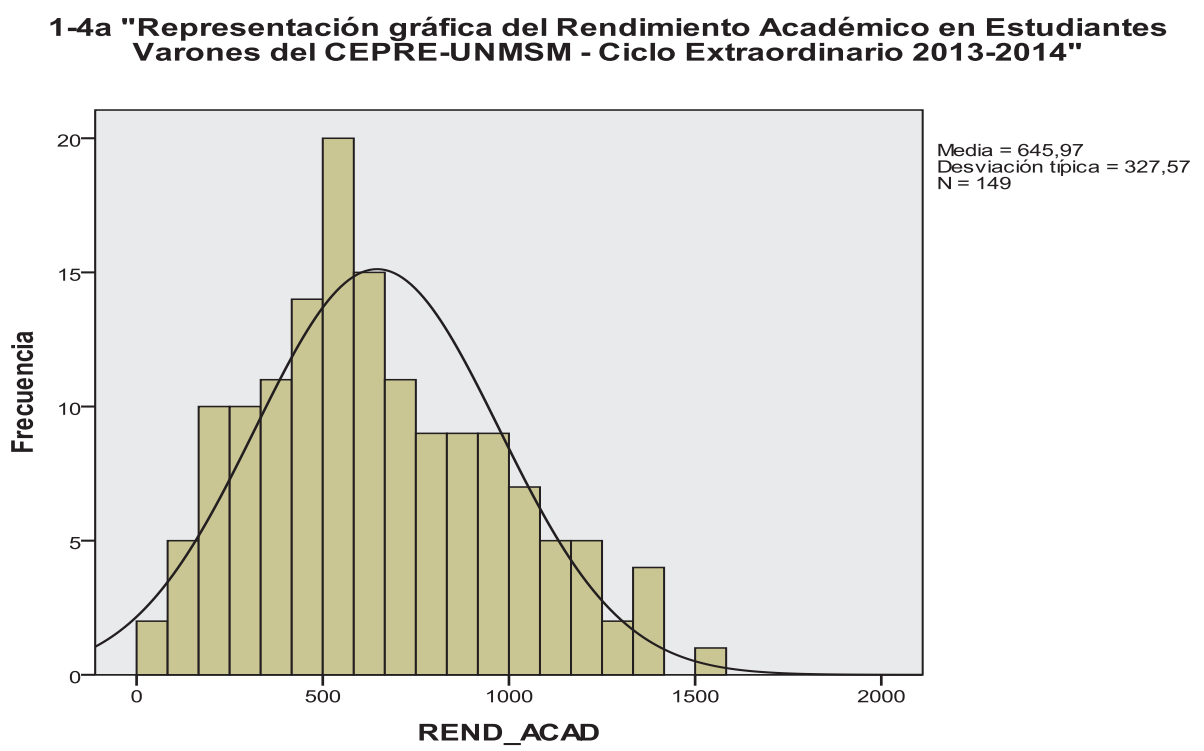

1-4b "Representación gráfica del Rendimiento Académico en Estudiantes Mujeres del CEPRE-UNMSM - Ciclo Extraordinario 2013-2014"

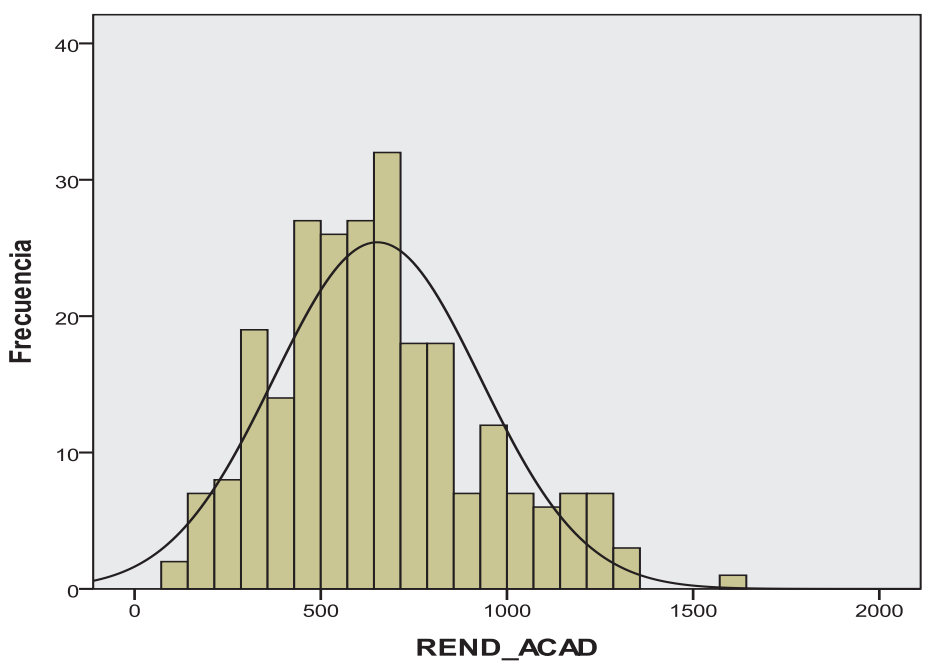

Media $=651,57$
Desviación típica $=278,019$ $\mathrm{N}=248$ 
Objetivo específico 2. Establecer la relación entre estrategias de aprendizaje y elección vocacional en los alumnos del CEPRE-UNMSM - Ciclo Extraordinario 2013-2014.

Hipótesis específica 1. Existe relación directa entre estrategias de aprendizaje y elección vocacional en los alumnos del CEPRE-UNMSM - Ciclo Extraordinario 2013-2014.

Hipótesis nula. No existe relación entre estrategias de aprendizaje y elección vocacional en los alumnos del CEPRE-UNMSM - Ciclo Extraordinario 2013 - 2014.

Hipótesis alternativa. Existe relación entre estrategias de aprendizaje y elección vocacional en los alumnos del CEPRE-UNMSM - Ciclo Extraordinario 2013-2014.

Considerando que las dos variables de estudio han sido medidas en escala nominal, para analizar la relación entre ambas variables usaremos el estadístico Chi -cuadrado de Pearson.

$\mathrm{H} 0$ = Las variables estrategias de aprendizaje y elección vocacional son independientes.

H1 = Las variables estrategias de aprendizaje y elección vocacional están relacionadas.

Pruebas de chi-cuadrado

\begin{tabular}{|c|c|c|c|}
\hline & Valor & gl & $\begin{array}{c}\text { Sig. asintótica } \\
\text { (bilateral) }\end{array}$ \\
\hline Chi-cuadrado de Pearson & $81,781 \mathrm{a}$ & 82 & 0,486 \\
\hline Razón de verosimilitudes & 86,580 & 82 & 0,343 \\
\hline No de casos válidos & 397 & & \\
\hline a. 104 casillas (82,5\%) tienen una frecuencia esperada inferior a 5. La frecuencia mínima esperada es 16. \\
\hline
\end{tabular}

De acuerdo con los resultados de Chi-cuadrado de Pearson ( $82.5 \%$ de casillas tienen una frecuencia esperada inferior a 5) y al $\mathrm{p}$-valor correspondiente $(\mathrm{p}=0.486)$ asumimos que las dos variables son independientes, no están asociadas, por lo tanto, no rechazamos la hipótesis nula. 
RELACIÓN ENTRE ESTRATEGIAS DE APRENDIZAJE, ELECCIÓN VOCACIONAL, RENDIMIENTO ACADÉMICO Y PERSONALIDAD PREDOMINANTE...

\begin{tabular}{|c|c|c|c|c|c|c|}
\hline & & & \multicolumn{3}{|c|}{ ESTRAT_DIM } & \multirow{2}{*}{ Total } \\
\hline & & & 1 & 2 & 3 & \\
\hline \multirow{2}{*}{ VOCACIÓN } & \multirow{2}{*}{1} & Recuento & 3 & 20 & 17 & 40 \\
\hline & & Frecuencia esperada & 6,3 & 15,3 & 18,3 & 40,0 \\
\hline & \multirow{2}{*}{2} & Recuento & 4 & 11 & 15 & 30 \\
\hline & & Frecuencia esperada & 4,8 & 11,5 & 13,8 & 30,0 \\
\hline & \multirow{2}{*}{3} & Recuento & 0 & 2 & 2 & 4 \\
\hline & & Frecuencia esperada & ,6 & 1,5 & 1,8 & 4,0 \\
\hline & \multirow{2}{*}{4} & Recuento & 1 & 0 & 0 & 1 \\
\hline & & Frecuencia esperada & ,2 &, 4 &, 5 & 1,0 \\
\hline & \multirow{2}{*}{5} & Recuento & 0 & 1 & 2 & 3 \\
\hline & & Frecuencia esperada &, 5 & 1,1 & 1,4 & 3,0 \\
\hline & \multirow{2}{*}{6} & Recuento & 0 & 1 & 2 & 3 \\
\hline & & Frecuencia esperada &, 5 & 1,1 & 1,4 & 3,0 \\
\hline & \multirow{2}{*}{7} & Recuento & 2 & 0 & 1 & 3 \\
\hline & & Frecuencia esperada &, 5 & 1,1 & 1,4 & 3,0 \\
\hline & \multirow{2}{*}{8} & Recuento & 0 & 0 & 1 & 1 \\
\hline & & Frecuencia esperada & ,2 &, 4 &, 5 & 1,0 \\
\hline & \multirow{2}{*}{9} & Recuento & 0 & 0 & 1 & 1 \\
\hline & & Frecuencia esperada & ,2 & ,4 &, 5 & 1,0 \\
\hline & \multirow{2}{*}{11} & Recuento & 1 & 3 & 6 & 10 \\
\hline & & Frecuencia esperada & 1,6 & 3,8 & 4,6 & 10,0 \\
\hline & \multirow{2}{*}{12} & Recuento & 5 & 11 & 8 & 24 \\
\hline & & Frecuencia esperada & 3,8 & 9,2 & 11,0 & 24,0 \\
\hline & \multirow{2}{*}{13} & Recuento & 4 & 7 & 15 & 26 \\
\hline & & Frecuencia esperada & 4,1 & 10,0 & 11,9 & 26,0 \\
\hline
\end{tabular}


Hilda Chávez Ch., Juan Morocho S., César Alvites R., José Vega G., Jesús Ruelas S., Christian Espinoza M., Juan Gómez Ch., Fabiana Santiago A.

\begin{tabular}{|c|c|c|c|c|c|}
\hline \multirow{2}{*}{14} & Recuento & 4 & 6 & 4 & 14 \\
\hline & Frecuencia esperada & 2,2 & 5,4 & 6,4 & 14,0 \\
\hline \multirow{2}{*}{15} & Recuento & 1 & 0 & 1 & 2 \\
\hline & Frecuencia esperada & ,3 &, 8 & ,9 & 2,0 \\
\hline \multirow{2}{*}{16} & Recuento & 1 & 0 & 0 & 1 \\
\hline & Frecuencia esperada & ,2 &, 4 &, 5 & 1,0 \\
\hline \multirow{2}{*}{17} & Recuento & 0 & 1 & 0 & 1 \\
\hline & Frecuencia esperada & ,2 &, 4 &, 5 & 1,0 \\
\hline \multirow{2}{*}{18} & Recuento & 0 & 1 & 1 & 2 \\
\hline & Frecuencia esperada & ,3 &, 8 & ,9 & 2,0 \\
\hline \multirow{2}{*}{19} & Recuento & 0 & 1 & 0 & 1 \\
\hline & Frecuencia esperada &, 2 &, 4 &, 5 & 1,0 \\
\hline \multirow{2}{*}{20} & Recuento & 0 & 1 & 1 & 2 \\
\hline & Frecuencia esperada & ,3 &, 8 & ,9 & 2,0 \\
\hline \multirow{2}{*}{21} & Recuento & 1 & 3 & 8 & 12 \\
\hline & Frecuencia esperada & 1,9 & 4,6 & 5,5 & 12,0 \\
\hline \multirow{2}{*}{22} & Recuento & 3 & 2 & 3 & 8 \\
\hline & Frecuencia esperada & 1,3 & 3,1 & 3,7 & 8,0 \\
\hline \multirow{2}{*}{23} & Recuento & 2 & 4 & 11 & 17 \\
\hline & Frecuencia esperada & 2,7 & 6,5 & 7,8 & 17,0 \\
\hline \multirow{2}{*}{24} & Recuento & 0 & 3 & 1 & 4 \\
\hline & Frecuencia esperada & ,6 & 1,5 & 1,8 & 4,0 \\
\hline \multirow{2}{*}{25} & Recuento & 0 & 1 & 0 & 1 \\
\hline & Frecuencia esperada & ,2 &, 4 & ,5 & 1,0 \\
\hline \multirow{2}{*}{26} & Recuento & 0 & 3 & 1 & 4 \\
\hline & Frecuencia esperada & ,6 & 1,5 & 1,8 & 4,0 \\
\hline
\end{tabular}


RELACIÓN ENTRE ESTRATEGIAS DE APRENDIZAJE, ELECCIÓN VOCACIONAL, RENDIMIENTO ACADÉMICO Y PERSONALIDAD PREDOMINANTE...

\begin{tabular}{|c|c|c|c|c|c|}
\hline \multirow{2}{*}{27} & Recuento & 0 & 2 & 2 & 4 \\
\hline & Frecuencia esperada & ,6 & 1,5 & 1,8 & 4,0 \\
\hline \multirow{2}{*}{28} & Recuento & 0 & 1 & 1 & 2 \\
\hline & Frecuencia esperada &, 3 & ,8 & ,9 & 2,0 \\
\hline \multirow{2}{*}{29} & Recuento & 3 & 3 & 1 & 7 \\
\hline & Frecuencia esperada & 1,1 & 2,7 & 3,2 & 7,0 \\
\hline \multirow{2}{*}{30} & Recuento & 8 & 23 & 18 & 49 \\
\hline & Frecuencia esperada & 7,8 & 18,8 & 22,5 & 49,0 \\
\hline \multirow{2}{*}{31} & Recuento & 0 & 1 & 0 & 1 \\
\hline & Frecuencia esperada & ,2 & ,4 &, 5 & 1,0 \\
\hline \multirow{2}{*}{32} & Recuento & 0 & 1 & 1 & 2 \\
\hline & Frecuencia esperada & ,3 & ,8 & ,9 & 2,0 \\
\hline \multirow{2}{*}{33} & Recuento & 0 & 0 & 1 & 1 \\
\hline & Frecuencia esperada & ,2 & ,4 & ,5 & 1,0 \\
\hline \multirow{2}{*}{34} & Recuento & 0 & 1 & 0 & 1 \\
\hline & Frecuencia esperada & ,2 & ,4 &, 5 & 1,0 \\
\hline \multirow{2}{*}{35} & Recuento & 14 & 24 & 40 & 78 \\
\hline & Frecuencia esperada & 12,4 & 29,9 & 35,8 & 78,0 \\
\hline \multirow{2}{*}{36} & Recuento & 2 & 0 & 2 & 4 \\
\hline & Frecuencia esperada & 6 & 1,5 & 1,8 & 4,0 \\
\hline \multirow{2}{*}{37} & Recuento & 0 & 1 & 1 & 2 \\
\hline & Frecuencia esperada & ,3 & ,8 & ,9 & 2,0 \\
\hline \multirow{2}{*}{38} & Recuento & 0 & 2 & 0 & 2 \\
\hline & Frecuencia esperada & ,3 &, 8 & ,9 & 2,0 \\
\hline \multirow{2}{*}{39} & Recuento & 3 & 5 & 3 & 11 \\
\hline & Frecuencia esperada & 1,7 & 4,2 & 5,0 & 11,0 \\
\hline
\end{tabular}




\begin{tabular}{|l|c|l|c|c|c|c|}
\hline & \multirow{3}{*}{40} & Recuento & 1 & 3 & 8 & 12 \\
\cline { 3 - 7 } & & Frecuencia esperada & 1,9 & 4,6 & 5,5 & 12,0 \\
\hline \multirow{2}{*}{41} & Recuento & 0 & 0 & 1 & 1 \\
\cline { 3 - 7 } & & Frecuencia esperada &, 2 &, 4 &, 5 & 1,0 \\
\hline \multirow{2}{*}{42} & Recuento & 0 & 2 & 2 & 4 \\
\cline { 3 - 7 } & & Frecuencia esperada &, 6 & 1,5 & 1,8 & 4,0 \\
\hline & 43 & Recuento & 0 & 1 & 0 & 1 \\
\cline { 3 - 7 } & & Frecuencia esperada &, 2 &, 4 &, 5 & 1,0 \\
\hline & Recuento & 63 & 152 & 182 & 397 \\
\hline & & Frecuencia esperada & 63,0 & 152,0 & 182,0 & 397,0 \\
\hline
\end{tabular}

Objetivo específico 3. Determinar la relación entre estrategias de aprendizaje y rendimiento académico en los alumnos del CEPRE-UNMSM - Ciclo Extraordinario 2013 - 2014.

Como una variable es cualitativa (estrategias de aprendizaje) y la otra variable es cuantitativa (rendimiento académico), se usará la t de Student para datos apareados o relacionados con el fin de encontrar la relación entre estas dos variables.

Hipótesis específica 2. Entre las estrategias de aprendizaje y el rendimiento académico de los alumnos del CEPRE-UNMSM - Ciclo Extraordinario 2013 - 2014 existe relación directa.

Hipótesis nula. No existe relación directa entre las estrategias de aprendizaje y el rendimiento académico de los alumnos del CEPRE-UNMSM - Ciclo Extraordinario 2013 - 2014.

Hipótesis alternativa. Existe relación directa entre estrategias de aprendizaje y rendimiento académico en los alumnos del CEPRE-UNMSM - Ciclo Extraordinario 2013 - 2014.

Considerando que la variable estrategias de aprendizaje tiene un nivel de medición nominal, mientras que la variable rendimiento académico tiene un nivel de medición de intervalo, para analizar la relación entre ambas variables utilizaremos el coeficiente Rho de Spearman. 


\begin{tabular}{|c|c|c|c|c|}
\hline \multicolumn{5}{|c|}{ Correlaciones } \\
\hline \multirow{2}{*}{$\begin{array}{c}\text { Rho de } \\
\text { Spearman }\end{array}$} & \multirow{3}{*}{ REND_ACAD } & REND_ACAD & ESTRAT_DIM \\
\cline { 1 - 3 } & & Coeficiente de correlación & 1,000 &, 044 \\
\cline { 3 - 5 } & & Sig. (unilateral) & $\cdot$ &, 190 \\
\cline { 3 - 5 } & \multirow{2}{*}{ ESTRAT_DIM } & Coeficiente de correlación &, 044 & 397 \\
\hline & & Sig. (unilateral) &, 190 & 1,000 \\
\hline & & $N^{0}$ & 397 & 397 \\
\hline
\end{tabular}

Como Rho $=0.044$ podemos apreciar que entre rendimiento académico y estrategias de aprendizaje la relación es insignificante, y que p-valor $=0.190$ nos indica independencia entre las variables. Podemos concluir que no existe relación entre ambas variables; son independientes.

Objetivo 4. Determinar la relación entre estrategias de aprendizaje y personalidad predominante en los alumnos del CEPRE-UNMSM - Ciclo Extraordinario 2013-2014.

Hipótesis específica 3. Existe relación directa entre estrategias de aprendizaje y personalidad predominante en los alumnos del CEPRE-UNMSM - Ciclo Extraordinario 2013-2014.

Como ambas variables tienen un nivel de medición nominal, entonces la relación se establece utilizando Chi-cuadrado.

\begin{tabular}{|l|c|c|c|}
\hline \multicolumn{5}{|c|}{ Pruebas de Chi-cuadrado } \\
\hline & Valor & gl & Sig. asintótica (bilateral) \\
\hline Chi-cuadrado de Pearson & $17,899 \mathrm{a}$ & 12 &, 119 \\
\hline Razón de verosimilitudes & 18,017 & 12 &, 115 \\
\hline Asociación lineal por lineal &, 001 & 1 &, 981 \\
\hline $\mathrm{N}^{\circ}$ de casos válidos & 397 & & \\
\hline a. 2 casillas $(9,5 \%)$ tienen una frecuencia esperada inferior a 5. La frecuencia mínima esperada es 2,54. \\
\hline
\end{tabular}

$\mathrm{H} 0=$ Las variables estrategias de aprendizaje y personalidad predominante son independientes.

H1 = Las variables estrategias de aprendizaje y personalidad predominante están relacionadas.

De acuerdo con los resultados de Chi-cuadrado de Pearson (9.5\% de casillas tienen una frecuencia esperada inferior a 5) y al $p$-valor correspondiente $(\mathrm{p}=0.119)$ asumimos que las dos variables son independientes, no están asociadas; por lo tanto, no rechazamos la hipótesis nula. 


\section{CONCLUSIONES Y RECOMENDACIONES}

1. Descripción de las estrategias de aprendizaje.- Las estrategias de aprendizaje más utilizadas por los alumnos del Ciclo Extraordinario 2013-2014 son: repetición y relectura $(80.88 \%)$, seguida de comprensión ( $78 \%$ ) y motivación intrínseca $(77.88 \%)$.

2. Asimismo, las estrategias de aprendizaje menos utilizadas por los alumnos son: control de ansiedad (63\%), selección y organización (63.75\%), y horario y plan de trabajo $(64.13 \%)$.

3. Descripción de la elección vocacional. Las carreras profesionales que tienen mayor demanda son: Medicina Humana (18.3\%), Ingeniería Industrial (13.5\%) y Administración (10.3\%). Son varias las carreras profesionales que tienen menor demanda, entre ellas mencionaremos a Literatura, Química y Sociología -entre otras-, cada una de ellas con una demanda del $0.2 \%$.

4. Descripción del rendimiento académico. El rendimiento académico promedio de los alumnos del Ciclo Extraordinario 2013-2014 del CEPRE - UNMSM es de 649.46 puntos, con una desviación estándar de 297.189. Al efectuar un análisis del rendimiento académico, considerando la variable sexo encontramos que las mujeres tienen un rendimiento académico promedio (651.57 puntos) mayor que el de los varones (645.97 puntos).

5. En correlación estrategias de aprendizaje y elección vocacional, de acuerdo con los resultados de la Chi-cuadrado de Pearson ( $82.5 \%$ de casillas tienen una frecuencia esperada inferior a 5) y al $p$-valor correspondiente $(p=0.486)$ asumimos que las dos variables son independientes, no están asociadas y, por lo tanto, no rechazamos la hipótesis nula.

6. En correlación estrategias de aprendizaje y rendimiento académico. Para analizar la relación entre ambas variables se utilizó el coeficiente Rho de Spearman. Como Rho $=0.044$ podemos apreciar que entre rendimiento académico y estrategias de aprendizaje la relación es insignificante y que p-valor $=0.190$ nos indica independencia entre las variables. Podemos concluir que no existe relación entre ambas variables; son independientes.

7. En correlación estrategias de aprendizaje y personalidad. De acuerdo con los resultados de Chi-cuadrado de Pearson $(9.5 \%$ de casillas tienen una frecuencia esperada inferior a 5) y al $p$-valor correspondiente $(p=0.119)$ asumimos que las dos variables son independientes, no están asociadas y, por lo tanto, no rechazamos la hipótesis nula.

8. Desde el punto de vista técnico-científico esta investigación contribuirá a enriquecer los conocimientos con relación a las variables de estudio. Por un lado, permitirá fortalecer la validez, confiabilidad y objetividad de los instrumentos psicométricos utilizados. Por otro lado, posibilita el desarrollo e implementación de programas de intervención a fin de estimular las carencias detectadas a nivel cognitivo (estrategias de aprendizaje), fortalecer la toma de 
decisiones con respecto a la carrera elegida, estableciendo la relación entre su personalidad predominante y la elección vocacional. Asimismo, el conocimiento objetivo de las variables estudiadas en función del tipo de institución educativa, tipo de familia y lugar de procedencia nos permitirá brindar la orientación correspondiente no solo a los alumnos del CEPRE-UNMSM, sino también sugerir algunos cambios o la implementación de estrategias correctivas a nivel de las instituciones educativas de Lima, por UGEL o proporcionar los alcances a las autoridades del Ministerio de Educación.

\section{REFERENCIAS}

De la Fuente, J. y Justicia, F. (2003). Escala de estrategias de aprendizaje ACRAabreviada, para alumnos universitarios. Revista electrónica de investigación psicoeducativa y psicopedagógica.

Aliaga, J. (1988). La inteligencia, la personalidad, la actitud y el rendimiento hacia la matemática. Lima: Universidad San Martín de Porres.

Aliaga, J. et al. (2001). Variables psicológicas relacionadas con el rendimiento académico en matemática y estadística en alumnos del primer y segundo año de la Facultad de Psicología de la UNMSM.

Apaza, Y. (1988). Las actitudes y hábitos de estudio de los alumnos ingresantes a los Institutos Superiores Pedagógicos Privados de Cusco, Cusco: UPAC.

Buendía, L. y Olmedo, E.M. (2003). Estudio transcultural de los enfoques de aprendizaje en educación superior. Revista de Investigación Educativa.21(2), 371-386.

Castillo, I., Balaguer, I. y Duda J.L. (2003). Las teorías personales sobre el logro académico y su relación con la alienación escolar. Psicothema, 15(1), 75-81.

Condolo, J. (2010). Inteligencia emocional y rendimiento académico de los alumnos del $5^{\circ}$ año de secundaria de la I.E. María Inmaculada-APREC del distrito de San Martín de Porres.

Mas Tous, C. y Medinas Amorós, M. (2007) Motivaciones para el estudio en universitarios. Anales de Psicología. 23(1), 17-24.

Miljanovich Costilla, M. (2000). Relaciones entre la inteligencia general, el rendimiento académico y la comprensión de lectura en el campo educativo. Tesis para optar el Grado de Doctor en Educación. Lima: Universidad Nacional Mayor de San Marcos.

Ponce, C. (1988). Estudio comparativo sobre los hábitos de estudio y su relación con la inteligencia y la personalidad en grupos de ingresantes a la Universidad 
Hilda Chávez Ch., Juan Morocho S., César Alvites R., José Vega G., Jesús Ruelas S., Christian Espinoza M., Juan Gómez Ch., Fabiana Santiago A.

Nacional Mayor de San Marcos. Tesis para optar el Grado de Doctor en Psicología. Lima: Universidad Nacional Mayor de San Marcos.

Reyes, Y. (2003). Relación entre el rendimiento académico, la ansiedad ante los exámenes, los rasgos de personalidad, el autoconcepto y la asertividad en estudiantes del primer año de Psicología de la UNMSM. Tesis.

Sánchez de Tagle, (2010). Rasgos de personalidad, inteligencia y rendimiento académico en estudiantes de la carrera de médico cirujano.

Villacorta, E. (2010). Inteligencia emocional y rendimiento académico en estudiantes de medicina humana de la Universidad Nacional de la Amazonía Peruana. 